\title{
Satellite Model for Network Simulator 3
}

\author{
Jani Puttonen \\ Magister Solutions Ltd \\ Jyväskylä, Finland \\ jani.puttonen@magister.fi \\ Janne Kurjenniemi \\ Magister Solutions Ltd \\ Jyväskylä, Finland \\ janne.kurjenniemi@magister.fi
}

\author{
Sami Rantanen \\ Magister Solutions Ltd \\ Jyväskylä, Finland \\ sami.rantanen@magister.fi \\ Kari Aho \\ Magister Solutions Ltd \\ Jyväskylä, Finland \\ kari.aho@magister.fi
}

\author{
Frans Laakso \\ Magister Solutions Ltd \\ Jyväskylä, Finland \\ frans.laakso@magister.fi \\ Guray Acar \\ European Space Agency \\ Noordwijk, The Netherlands \\ guray.acar@esa.int
}

\begin{abstract}
Digital Video Broadcasting (DVB) organisation has published a bluebook for the 2nd generation of satellite return link specification, DVB-RCS2. Also, the evolution of DVB-S2, currently called DVB-Sx, is in standardization process. Simulations have increased importance in standardization support as well as in internal product development due to the ability to provide faster feedback on technological solutions without the need for early-stage testbed and real systems development. However, there is a need for modular open-source satellite system simulator which would adopt the latest DVB satellite standards. Current simulators owned by satellite vendors, operators and research organizations may be proprietary, generally tailored for special purposes and/or may be based on commercial licenses. In this article we present the building blocks and architecture of satellite model developed on top of a widely used opensource system/packet level simulator Network Simulator 3 (NS-3). The satellite extensions are based on DVB-S2 and DVB-RCS2 specifications for forward and return links, respectively. Simulator module is developed to be highly modular and flexible to be able to match the future R\&D needs of satellite system vendors, operators, research organizations, as well as scientific community.
\end{abstract}

\section{Categories and Subject Descriptors}

I.6.5 [Simulation and Modeling]: Model DevelopmentModeling Methodologies; I.6.7 [Simulation and Modeling]: Simulation Support Systems - Environments

\section{General Terms}

Simulation, Modeling, Algorithm, Design, Performance

\section{Keywords}

Satellite, DVB-S2, DVB-RCS2, Network Simulator 3, NS-3

Permission to make digital or hard copies of all or part of this work for personal or classroom use is granted without fee provided that copies are not made or distributed for profit or commercial advantage and that copies bear this notice and the full citation on the first page. To copy otherwise, to republish, to post on servers or to redistribute to lists, requires prior specific permission and/or a fee.

Simutools 2014, March 17-19, Lisbon, Portugal

Copyright (C) 2014 ICST 978-1-63190-007-5

DOI 10.4108/icst.simutools.2014.254631

\section{INTRODUCTION}

European Telecommunications Standards Institute (ETSI) has standardized DVB-S2 [4] in 2005. The main use cases for DVB-S2 has been different broadcast services, interactive broadband applications and Digital Satellite News Gathering (DSNG). Currently, there is work going related to DVBSx [9], which is planned to be the successor of DVB-S2 with e.g. more specified MODulation and CODing Schemes (MODCODs). Also, Digital Video Broadcasting organisation has published a bluebook for 2 nd generation interactive satellite return channel specifications DVB-RCS2 [7]. Both DVB-S2 and DVB-RCS2 will improve the competitiveness of satellite technologies in offering modern IP-based services to customers.

However, DVB-S2 and DVB-RCS2 specifications leave quite many algorithms up to implementation; esp. higher layer Radio Resource Management (RRM) algorithms, such as scheduling and Adaptive Coding and Modulation (ACM). On the other hand, simulations have increased importance in standardization support as well as in internal product development due to the ability to provide faster feedback on technological solutions without the need for early-stage testbed and real systems development.

There do not exist proper publicly available DVB-S2/DVBRCS2 satellite system simulators. The simulators owned by satellite vendors, operators and research organizations may be proprietary, generally tailored for special purposes and/or may be based on commercial licenses. The Open Source Satellite Simulator (OS3) [10] is quite recently developed satellite simulator by the Technical University of Dortmund. It is based on OMNet++ and complementing the INET framework, and claims to be modular and user friendly. However, the focus has been on satellite mobility, satellite constellations, inclusion of wheather data and channel models, and not on satellite communication protocols. A DVB-RCS model has been developed at the University of Aberdeen [3] on top of Network Simulator 2 (NS-2). However, it is clearly lacking some essential features, and it does not provide DVB-RCS2 or DVB-S2 models. In addition, it is based on NS-2, which has problems related to scalability, modularity, maintenance, etc. In addition to simulators, satellite vendors often use emulators, e.g. for protocol testing. One open source example is OpenSAND [12][1], developed by Thales Alenia Space, which emulates DVB-RCS and DVB-S2.

The objective of this article is to describe development 
Table 1: Position parameters

\begin{tabular}{l|l|l|l}
\hline Id & Latitude $[\mathrm{deg}]$ & Longitude [deg] & Altitude $[\mathrm{km}]$ \\
\hline \hline GW 1 & 51.50 & -0.13 & 0.0 \\
GW 2 & 59.91 & 10.75 & 0.0 \\
GW 3 & 40.42 & -3.70 & 0.0 \\
GW 4 & 41.90 & 12.48 & 0.0 \\
GW 5 & 37.98 & 23.73 & 0.0 \\
GEO SAT & 0.0 & 33.0 & 35786.0 \\
\hline
\end{tabular}

of a packet-level Satellite Network Simulator (SNS) model on top of Network Simulator 3 (NS-3)[11] platform. NS-3 is an open source discrete-event simulator platform aimed to enable modern networking research on both IP and nonIP based networks. NS-3 was selected as a simulation platform, because it is highly modular, widely used and is proven to be scalable and fast compared to other network simulators, according to [13]. In addition, NS-3 includes many other both wireless access and wired technology models enabling multi-technology use cases. NS-3 is also based on non-commercial General Public License version 2 (GPLv2). SNS is designed to be modular and flexible so that it can be used to simulate different interactive transparent geostationary (GEO) satellite networks with minimal source code modifications. Adopted reference system scenario includes an interactive broadband satellite network with a multispot beam GEO satellite with a transparent payload and it adopts ETSI DVB-S2 [4] and DVB-RCS2 [7] specifications as the communication standards on the forward and the return links. RRM algorithms have been designed in parallel to the early architectural definitions of the simulator, thus the framework provides good baseline that can be later used for designing more optimal RRM solutions. It is expected that the flexible and modular architecture of the simulator will reduce the cumulative code development efforts throughout different parties, such as European Space Agency (ESA), when conducting their R\&D on satellite systems.

Section 2 presents the adopted satellite reference system. In Section 3 the simulator satellite module architecture is presented. Section 4 presents an initial results of simulator link budget verification. Finally, Section 5 summarizes the article.

\section{SELECTED REFERENCE SYSTEM}

As a reference system scenario, a single multi spot-beam satellite at geostationary orbit at about $35786 \mathrm{~km}$ altitude is considered. The system coverage is Europe using a single satellite located at 33 degrees East using Ka-band frequency (from 26.5 to $40.0 \mathrm{GHz}$ ) on the feeder and user links. The user link coverage consists of 72 spot-beams, which are illustrated with transmission antenna gain patterns in Figure 1. The user beams are served by 5 gateways with the positions shown in Fig. 1 and Tab. 1.

Frequency plan in both user and feeder links are illustrated in Fig. 2. The system allocates $2 \mathrm{GHz}$ to the feeder link: from $27.5 \mathrm{GHz}$ to $29.5 \mathrm{GHz}$ to uplink and from 17.7 $\mathrm{GHz}$ to $19.7 \mathrm{GHz}$ downlink. Different polarizations (Right Hand Circular Polarization (RHCP), Left Hand Circular Polarization (LHCP)) are assumed for feeder uplink and downlink. Full frequency re-use (reuse 1) is assumed in the feeder link, which means that each GW uses the same $2 \mathrm{GHz}$ band

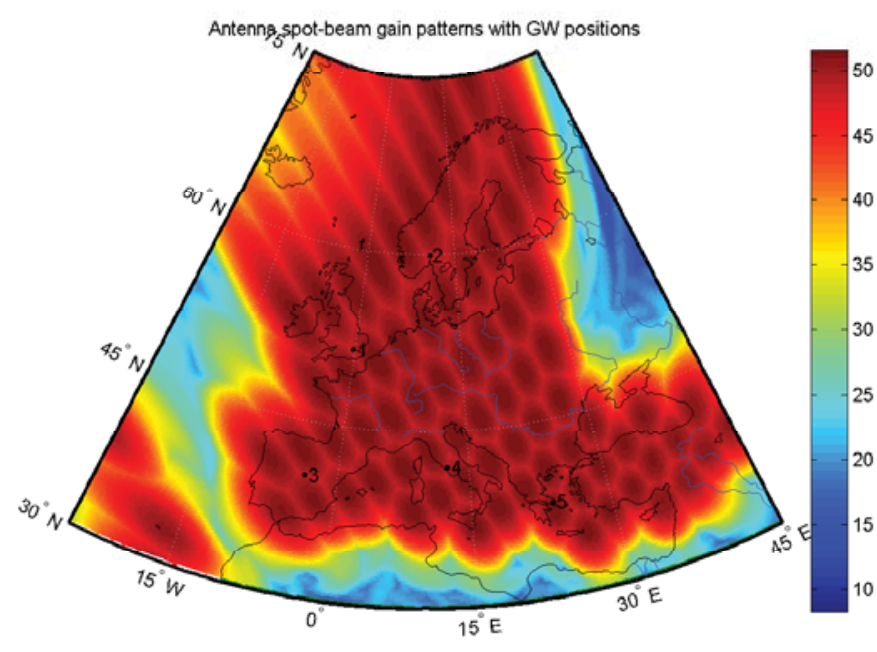

Figure 1: Antenna spot-beam antenna gain patterns

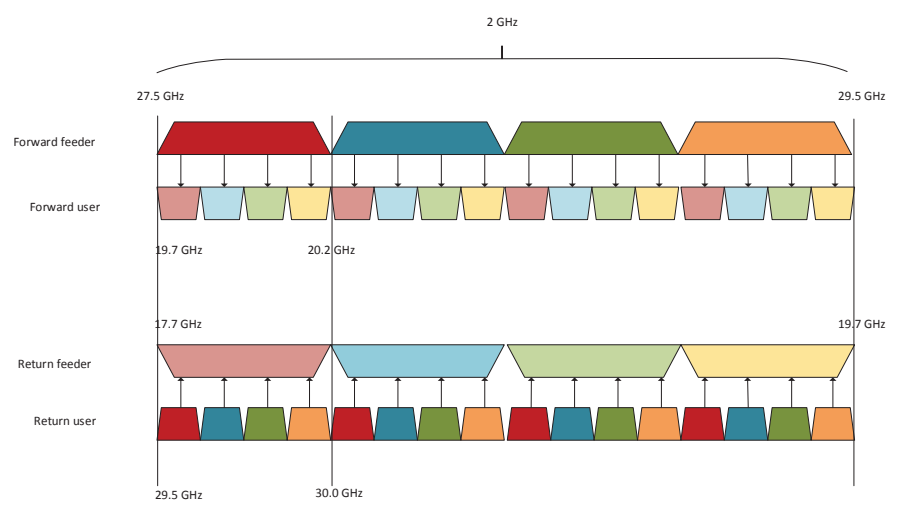

Figure 2: Frequency plan

on the feeder uplink; as well as in feeder downlink.

The system allocates $500 \mathrm{MHz}$ to the user link: from 29.5 $\mathrm{GHz}$ to $30 \mathrm{GHz}$ to uplink and from $19.7 \mathrm{GHz}$ to $20.2 \mathrm{GHz}$ to downlink. The $500 \mathrm{MHz}$ user band is divided into four $125 \mathrm{MHz}$ bands (i.e. four color re-use). Each spot-beam is allocated a $125 \mathrm{MHz}$ band on the user uplink, and another $125 \mathrm{MHz}$ band on the user downlink. Each GW can support an aggregate traffic to/from 16 beams $(2 \mathrm{GHz} / 125 \mathrm{MHz}$ $=16$ ). Thus, each GW is mapped to 16 beams. Since, the system comprises of 72 spot-beams in total, five GWs are needed to support them.

Return link uses a Multi-Frequency Time Division Multiple Access (MF-TDMA) which is composed of superframe sequences, superframes, frames, time slots and Bandwidth Time Units (BTUs) [7]. The used frame structures are dynamically configured by the Network Control Center (NCC) by using Superframe Composition Table (SCT), Frame Composition Table version 2 (FCT2) and Broadcast Composition Table $(B C T)$. The simulator does not explicitly model SCT, FCT and BCT, but the frame configurations may be changed by simulator parametrization. An example of the frame structures is presented in section 5.2.3. of DVB-RCS2 guidelines [6]. However, NCC models Terminal Burst Time Plan v2 (TBTPv2) and thus is capable of configuring the time slots dynamically for each superframe (e.g. start times, 


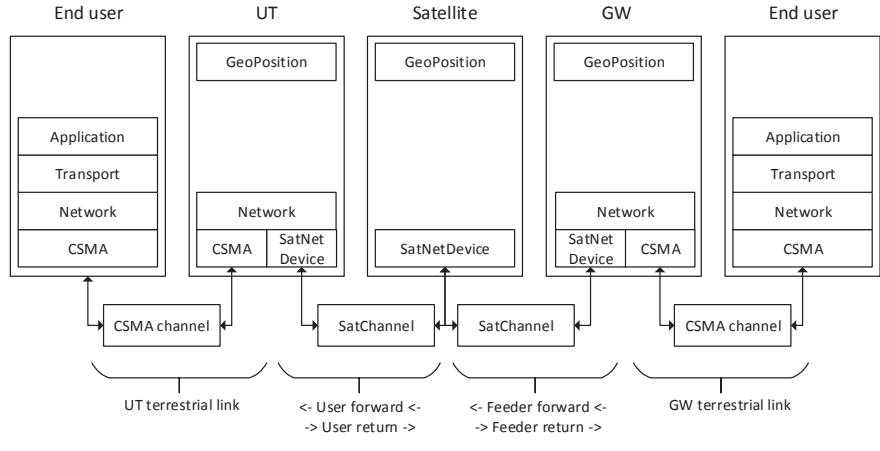

Figure 3: General end-to-end architecture

durations, waveforms).

Forward link is using DVB-S2 Time Division Multiplexing (TDM) structure which is described in [4] Section 5. The forward feeder link $2 \mathrm{GHz}$ bandwidth is divided into 16125 $\mathrm{MHz}$ carriers, where each carrier is statically mapped to a user link frequency color of $125 \mathrm{MHz}$ for a certain beam.

Note, that the simulator adopts a NS-3 attribute system, external configuration files, and a helper structure to support easy modification and adaptation of the reference system and its parameters.

\section{MODULE STRUCTURE}

\subsection{General}

Satellite model structure captures all the necessary functionality to be able to simulate multi-spot beam interactive transparent GEO satellite networks. This will entail modules for User Terminal (UT), Satellite (SAT), Gateway $(G W)$, Network Control Center (NCC), terrestrial nodes (end users) and their interaction (see Fig. 3).

In general, all satellite nodes require a new implementation of a NetDevice (SatNetDevice), which is inherited from NetDevice class. SatNetDevice implements Logical Link Control (LLC), Medium Access Control (MAC) and Physical (PHY) classes specific for each node. In addition, a new implementation of the Channel class (SatChannel) is needed for it to support satellite system received signal power calculations. In terrestrial link, the used access technology (behind UTs or GWs) may be anything supported by the NS-3 simulator, e.g. point-to-point, CSMA, WiFi. NCC is modeled as a shared module with all GW nodes.

Simulator implements both spherical and geodetic coordinate systems (WGS80 and GRS84) (latitude, longitude, altitude) in addition to the default Cartesian coordinate system. New coordinate system is needed for satellite domain nodes (UT, GEO satellite and GW). Note, that while node mobility is also supported, designing mobility models has not been the primary focus.

\subsection{User Terminal}

The UT architecture is presented in Fig. 4. The DVBRCS2 transmission chain is described on the left hand side and DVB-S2 reception chain on the right hand side. Incoming IP packets from IP layer are first classified to a set of IP packet queues in LLC layer. LLC holds a Return Link Encapsulator (RLE) entity, which holds the packet queues, but is also responsible of just-in-time fragmentation, encapsula- tion and packing to form a PHY packet, which fits into the time slot with given waveform (burst length, MODCOD). The scheduled time slots are signalled with TBTP messages from NCC.

Classification may be based on DiffServ Code Point (DSCP) and may represent e.g. different end users or end user application Quality of Service. Each queue is capable of collecting statistics from both incoming and outgoing packets/bytes for Request Manager ( $R M)$, which estimates the needed resources for each packet queue and requests resources with Capacity Request (CR) messages with certain Capacity Category (CC) from NCC; i.e. Rate-Based Dynamic Capacity (RBDC), Volume-Based Dynamic Capacity $(V B D C)$. Constant Rate Assignment (CRA) is modeled by simulator parametrization. Each IP packet queue is mapped to a separate Request Class $(R C)$ within the RM, thus the $\mathrm{RC}$ index is used as a queue/RC identifier in the sent $\mathrm{CRs}$ and received TBTPs. The used CCs, and their possible combinations, for each queue may be configured by simulator parameters.

UT scheduler is responsible for scheduling the transmission opportunities to LLC layer at the time of allocated time slots. At PHY layer, the packet is sent within a proper MFTDMA carrier and time slot to configured SatChannel instance. All the transmission related information are added to a SatSignalParameters (e.g. transmission power, carrier id), which is passed to the channel together with the transmitted PHY packet.

In DVB-S2 receiver at the UT, there are separate SatPhyRxCarrier classes handling packet receptions from a specific carrier. At SatPhyRx layer the SatSignalParameters content are checked and the packet is forwarded for actual reception to proper SatPhyRxCarrier instance. Each SatPhyRxCarrier has its own interference model instance, which is modeling co-channel interference; in either perpacket fashion by tracking all the co-channel transmissions, or by using interference traces. The SatPhyRxCarrier calculates user or feeder link SINR based on the received signal power, calculated by the channel itself, noise and interference, and maps the SINR into a error probability. Note, that the amount of SatPhyRxCarrier instances are depending on the carrier configuration in either forward or return direction.

At MAC layer the BB frames are disassembled and packets intended for the UT are forwarded to LLC. At the LLC layer, there is a Generic Stream Encapsulator (GSE) entity defragmenting and decapsuling the received GSE packets.

\subsection{Satellite}

The GEO satellite is presented in Fig. 5. Note, that the simulator supports currently transparent ("bent-pipe") payload, where user and feeder links are directly mapped to each other. Satellite only amplifies the signal without any packet processing. In principle, this means that satellite has a total of 72 PHY layers towards user link and 72 PHY layers towards feeder link, and at MAC layer they are directly switched to each other in one-to-one fashion. Each PHY is handling the transmissions and receptions in one single spot-beam (in either user or feeder link). The satellite receiver structure is similar to UT and GW, since it also has SatPhyRxCarrier structure and interference models to calculate the interference in either user or forward link. Note, that GEO satellite calculates also SINR, because the two 


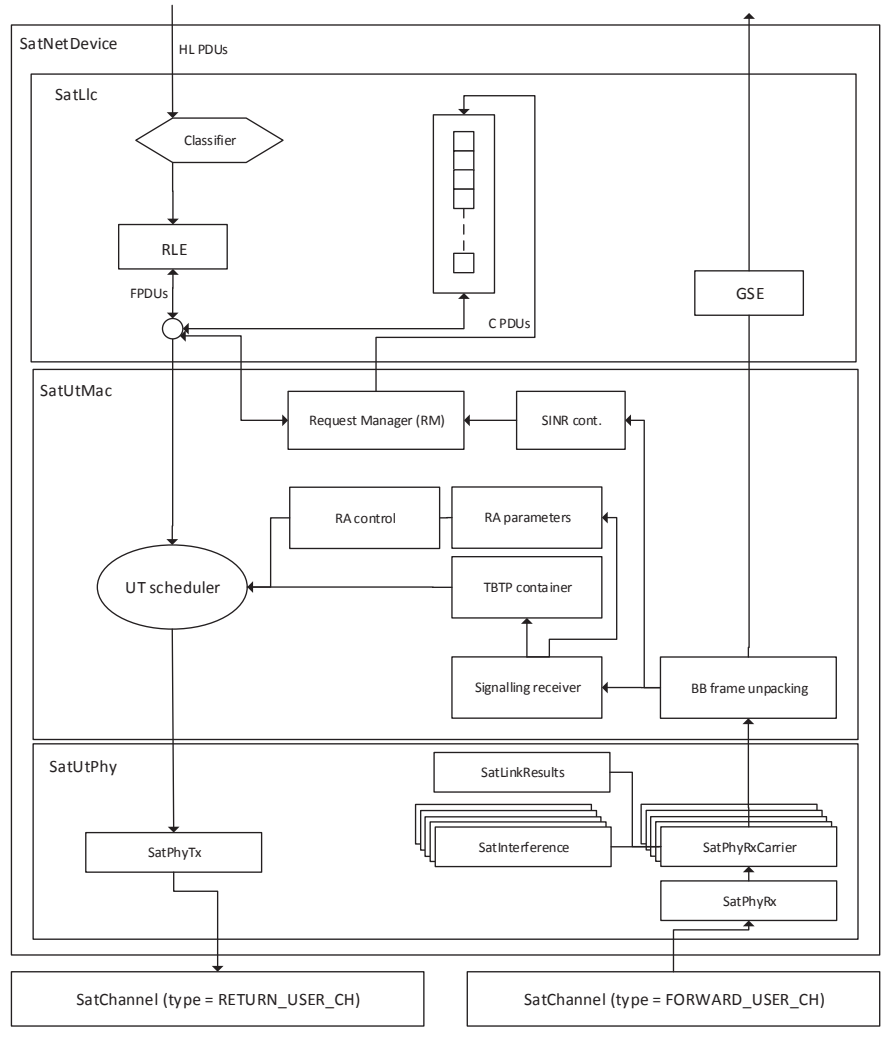

Figure 4: UT architecture

phase SINR calculation has been adopted. SINR is calculated separately for user and feeder links and combined by a composite SINR equation at the final receiver [8].

\subsection{Gateway}

The GW architecture is presented in Fig. 6. The DVB$\mathrm{S} 2$ transmission chain is described on the left hand side and DVB-RCS2 reception chain on the right hand side. Architecture of the SatNetDevice of GW is in general quite similar to UT architecture. However, a major difference is that GW has as many SatNetDevices as there are spot-beams served by the GW, thus one SatNetDevice is serving all the UTs within one spot-beam. The maximum number of SatNetDevices in the current reference system is 16 , since the total of $2 \mathrm{GHz}$ feeder link bandwidth is divided into $16125 \mathrm{MHz}$

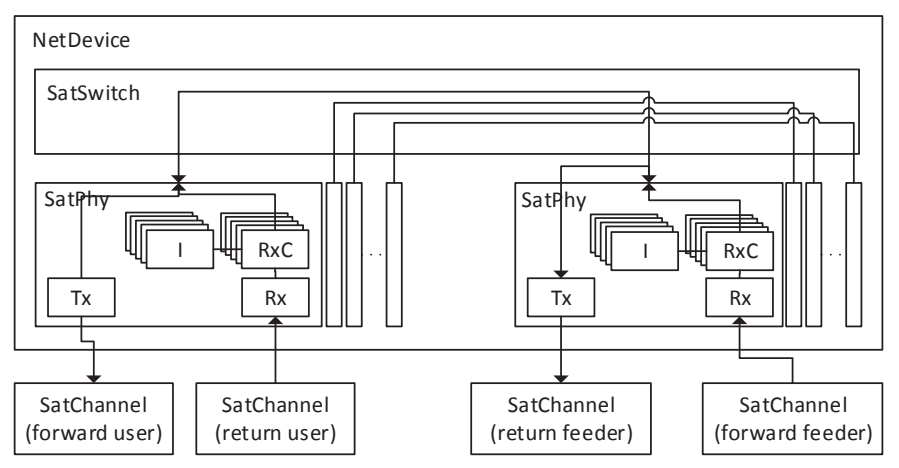

Figure 5: Satellite architecture

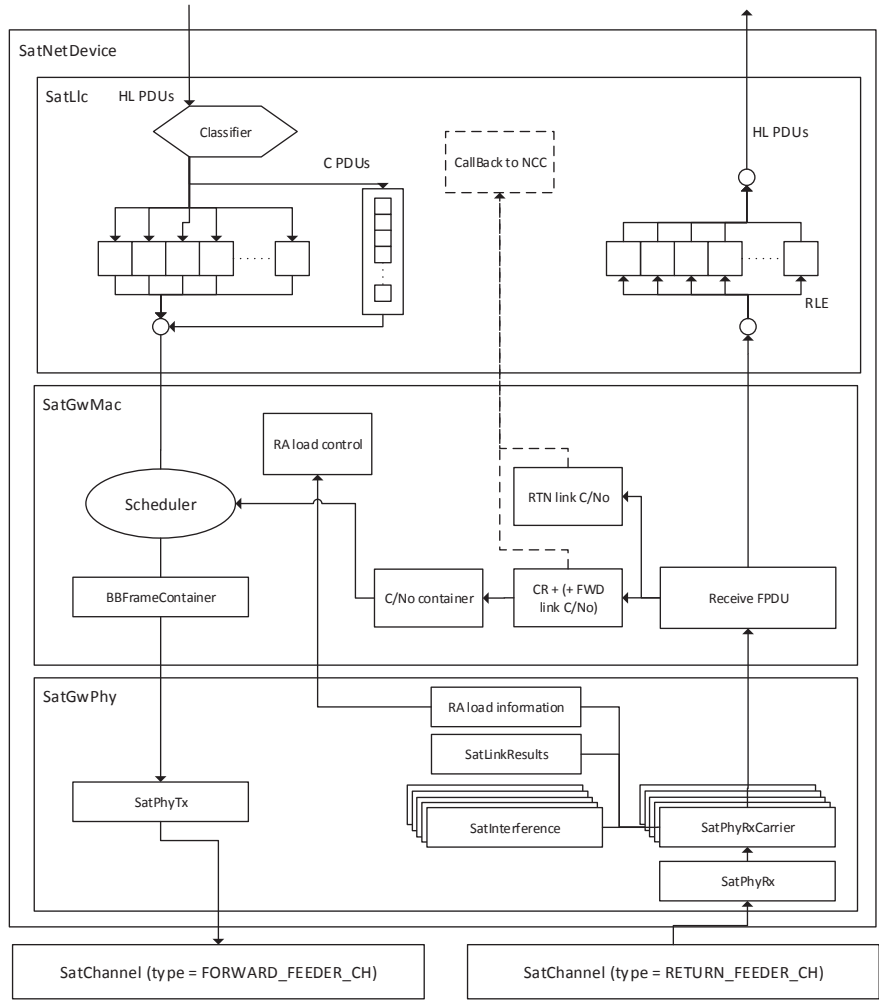

Figure 6: GW architecture

carriers.

The GW's LLC entity has one GSE entity per attached UT. The classifier classifies the packets based on DSCP, but also based on destination MAC address to separate GSE entities. DVB-S2 transmitter is constantly transmitting Baseband Frames (BBFrames), where each BBFrame holds higher layer packets with only one MODCOD. The length of a $B B$ Frame may be either 16200 or 64800 coded bits, thus the duration to transmit a BBFrame varies based on MODCOD. If the GW does not have any data to transmit, it is generating dummy frames. The DVB-RCS2 receiver is capable of receiving both dedicated access and Random Access (RA) slots. Otherwise, the reception logic is similar to UT architecture.

NCC is not implemented as a separate node, but as a shared module between GWs. This approach does not necessitate a communication protocol design/model between GW and NCC. Simulator implements one NCC module, which has a separate return link scheduler for each spot-beam. The same NCC is shared by all GWs, and the communication between them (e.g. CRs, TBTPs) is handled with NS-3 callback functions. This enables easy modification of the approach at later stages, if needed.

\subsection{Channel}

The simulator channel implementation (SatChannel) maps into a frequency color (bandwidth). The main purpose of a channel is to be able to pass packets within a one frequency band to all receivers sharing the same bandwidth. In other words, all the co-channel beams are sharing the same channel, and beams in different frequency bands are fully separated to different channel instances. 
In user link, there are a total of four channel instances (SatChannel) per direction, each representing one $125 \mathrm{MHz}$ bandwidth. In the used reference system, there are a total of $72 / 4=18$ spot-beams sharing the same user link channel. Thus, UTs within the 18 spot-beams sharing the same channel shall be able to interfere each other. In feeder link, there are a total of 16 channel instances per direction $(2 \mathrm{GHz} /$ $0.125 \mathrm{MHz}$ ), each representing one $125 \mathrm{MHz}$ bandwidth. All GWs are sharing the same frequency band, thus there may be a maximum of $5 \mathrm{GWs}$ sharing the same channel instance.

The physical layer of the GW and UT nodes and the channel structure is presented in Fig. 7. The figure illustrates e.g. the transmission from GEO satellite to a either UT or GW. SatChannel is an object which enables packet transmissions and received signal power calculation from a node to another within the same frequency band (color). Every receiving node has been registered to the same SatChannel, which are sharing the same frequency band (intra-beam + co-channel beam). In addition, SatChannel is responsible for calculating the received signal power for each receiver, regardless whether the receiver is the actual destination of the packet. The structure enables the packet-by-packet interference calculation (in all links), because the packet-bypacket interference model at the receiver is receiving all the co-channel transmissions with the calculated received power.

To enable the received signal power calculation, SatChannel needs:

- Mobility model of both transmitter and receiver for node coordinates

- Packet transmission related information (e.g. Equivalent Isotropically Radiated Power (EIRP)) passed within SatSignalParameters

- Free Space Loss (FSL) module to calculate propagation loss

- Fading information of the terrestrial node, which is acquired from the SatPhyRx / SatPhyTx layer

- Satellite antenna gain patterns ( $\mathrm{Rx}$ in uplink, $\mathrm{Tx}$ in downlink) are needed to be able to calculate the antenna gain at terrestrial node position

- Possibly external channel trace files

In addition, the SatChannel has a separate module for calculating the propagation delay between the transmitter and receiver positions. The propagation delay model is slant range dependent with signal propagation velocity of speed of light. This enables the realistic propagation delay modeling of the satellite link. However, note that this necessitates also a timing advance model to account for different slant ranges of UTs.

At the receiver, the SatPhyRxCarrier object calculates the link SINR using the received signal power, interference from the interference model and noise. The composite SINR is calculated by combining the user and feeder link SINRs. Packet errors are modeled by mapping the composite SINR to PER according to SINR to PER mapping tables [2]. E.g. the [5] holds Es/No to PER mapping tables for DVB-S2.

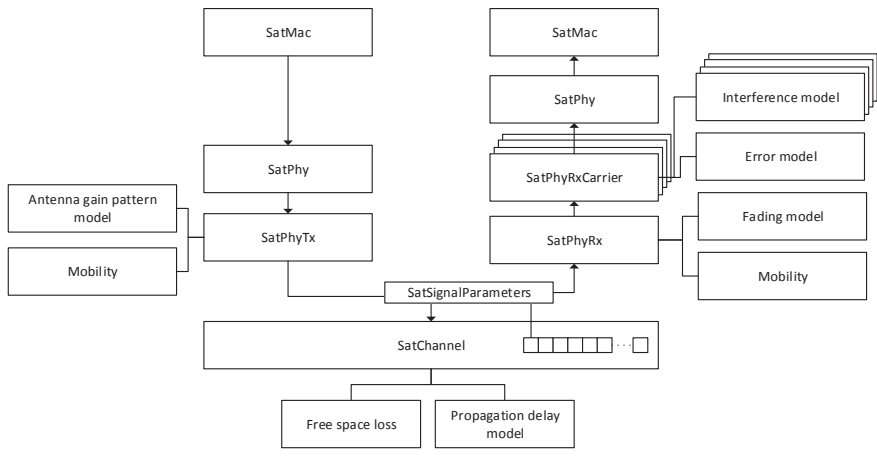

Figure 7: Physical layer and channel structure

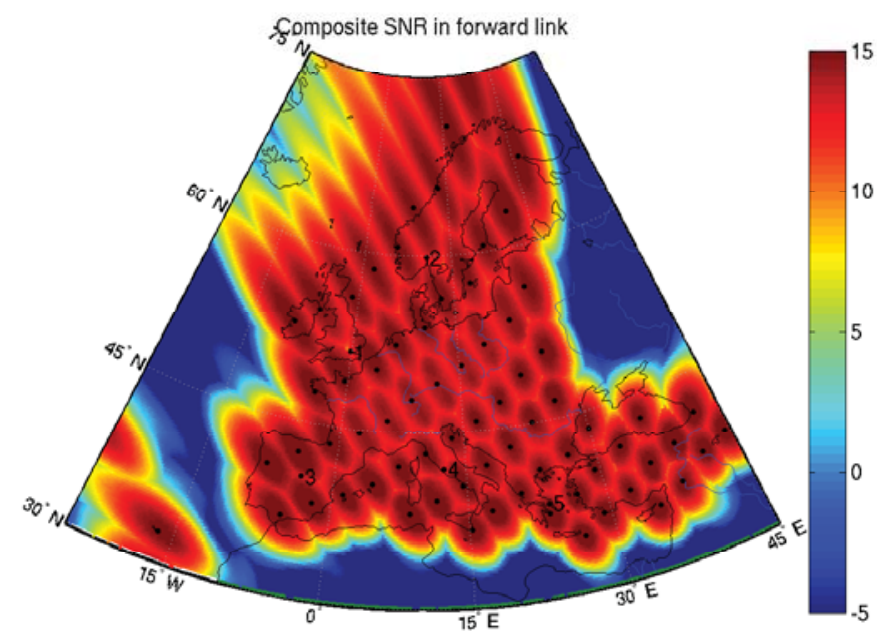

Figure 8: Composite SINR in forward link

\section{VERIFICATION}

In this section, the composite SNR for forward link at all possible UT positions have been calculated with Matlab by using the link budget parameters presented in Tab. 2. Calculations take into account in both user and feeder links, $\mathrm{Tx} / \mathrm{Rx}$ antenna gain patterns and antenna losses, EIRP, slant range dependent free space loss, noise and interference. Note, that the DVB-S2 based forward link assumes constant interference per UT position due to dummy frames. Fig. 8 shows the composite SINR for each position. When compared to the link results presented at [5], the link closes in the whole coverage area. These results may be used for link budget parameterization in the actual satellite model.

\section{CONCLUSION}

In this article we have presented a satellite model for Network Simulator 3 (NS-3), which is a modular open-source packet and system level simulator. The satellite model implements ETSI DVB-S2 in the forward link and DVB-RCS2 in the return link. The focus in this article has been in general simulator building blocks: module architecture, selected reference system, channel structures and models and physical layer modeling. The configured reference system link budget has been initially verified with Matlab. Satellite module is developed to be highly modular and flexible to be able to match the future $R \& D$ needs of satellite system ven- 
dors, operators, research organizations, as well as scientific community. Research topics may be related to e.g. overall satellite system performance analysis, RRM protocol optimization, regenerative and adaptive payload. The satellite module source code has been planned to be integrated NS-3 main release for public access after completion.

\section{ACKNOWLEDGEMENTS}

This project have been funded by European Space Agency in the scope of ARTES 5.1 AO6947 "Development of an open-source, modular and flexible satellite network simulator" project (contract no: 4000106081/12/NL/NR).

\section{REFERENCES}

[1] C. Baudoin et al. Overview of Platine emulation testbed and its utilization to support DVB-RCS/S2 evolutions. 5th Advanced satellite multimedia systems conference (ASMA) and the 11th signal processing for space communications workshop (spsc), September 2010.

[2] K. Brueninghaus et al. Link Performance Models for System Level Simulations of Broadband Radio Access Systems. IEEE International Symposium on Personal, Indoor and Mobile Radio Communications, 2005.

[3] DVB-RCS(2) for ns-2. MFTDMA-DAMA, http://homepages.abdn.ac.uk/r.secchi/pages/dvbrcs_ns2.htm, referenced 10.1.2014.

[4] Digital Video Broadcasting (DVB); Second generation framing structure, channel coding and modulation systems for Broadcasting, Interactive Services, News Gathering and other broadband satellite applications (DVB-S2), 2005.

[5] Digital Video Broadcasting (DVB); User guidelines for the second generation system for Broadcasting, Interactive Services, News Gathering and other broadband satellite applications (DVB-S2), 2005.

[6] Digital Video Broadcasting (DVB); Second Generation DVB Interactive Satellite System (DVB-RCS2); Guidelines for Implementation and Use of LLS, 2012.

[7] Digital Video Broadcasting (DVB); Second Generation DVB Interactive Satellite System (DVB-RCS2); Part 2: Lower Layers for Satellite standard, 2012.

[8] G. Maral et al. Satellite Communications Systems: Systems, Techniques and Technology. Wiley, 5th edition edition, 2010.

[9] A. Morello. DVB-Sx: The evolution of the (satellite systems) species. 31ST AIAA International Communications Satellite Systems Conference, October 2013.

[10] B. Niehoefer et al. The CNI Open Source Satellite Simulator based on OMNeT++. Proceedings of the 6th International ICST Conference on Simulation Tools and Techniques, March 2013.

[11] NS-3 Consortium. Network Simulator 3, http://www.nsnam.org, referenced 29.10.2013.

[12] OpenSAND. A satellite telecommunication system emulation platform, http://opensand.org/, referenced 10.1.2014.

[13] E. Weingartner. A Performance Comparison of Recent Network Simulators. IEEE International Conference on Communications, June 2009.
Table 2: Link budget parameters

\begin{tabular}{|c|c|}
\hline Parameter & Value \\
\hline \multicolumn{2}{|l|}{ UT } \\
\hline$\overline{P_{t e r m, t x}^{n, m a x}}$ & $45.2 \mathrm{dBW}$ \\
\hline$G_{\text {term,tx }}^{n, \max }$ & $4.0 \mathrm{~dB}$ \\
\hline$L_{\text {term }, t x}^{o u t}$ & $0.5 \mathrm{~dB}$ \\
\hline$L_{\text {term }, t x}^{\text {pointing }}$ & $\mathrm{dB} 1.0$ \\
\hline$L_{t e r m, t x}^{O B O}$ & $0.5 \mathrm{~dB}$ \\
\hline$G_{\text {term }}^{\max }$ & $41.7 \mathrm{~dB}$ \\
\hline$T_{\text {term }}$ & $290 \mathrm{~K}$ \\
\hline$\left(\frac{C}{I}\right)_{\text {fwd,dwn }}^{\text {othersys }}$ & $24.7 \mathrm{~dB}$ \\
\hline \multicolumn{2}{|l|}{ Satellite user } \\
\hline$P_{s a t}^{f w d, d w n}$ & $15.0 \mathrm{dBW}$ \\
\hline$G_{u S a t, t x}^{b_{n}}\left(\vartheta_{n}, \varphi_{n}\right)$ & $\begin{array}{l}\text { Acquired from an- } \\
\text { tenna patterns based } \\
\text { on terrestrial node } \\
\text { position (see Fig. } 1 \text { ) }\end{array}$ \\
\hline$L_{\text {sat }, \text { out }}^{f w d, d w n}$ & $2.85 \mathrm{~dB}$ \\
\hline$L_{\text {sat,ant }}^{f w d, d w n}$ & $1.0 \mathrm{~dB}$ \\
\hline$G_{u S a t, r x}^{b_{n}}\left(\vartheta_{n}, \varphi_{n}\right)$ & $\begin{array}{l}\text { Acquired from an- } \\
\text { tenna patterns based } \\
\text { on terrestrial node } \\
\text { position (see Fig. } 1 \text { ) }\end{array}$ \\
\hline$L_{\text {sat }, \text { ant }}^{r n t, u p}$ & $1.0 \mathrm{~dB}$ \\
\hline$T_{\text {sat }, \text { user }}$ & $691.83 \mathrm{~K}$ \\
\hline$\left(\frac{C}{I}\right)_{r t n, u p}^{A C I}$ & $17.0 \mathrm{~dB}$ \\
\hline$\left(\frac{C}{I}\right)_{r t n, u p}^{\text {othersys }}$ & $27.5 \mathrm{~dB}$ \\
\hline \multicolumn{2}{|l|}{ Satellite feeder } \\
\hline$P_{\text {sat }}^{r t n, d w n}$ & $-10.4 \mathrm{dbW}$ \\
\hline$G_{f S a t, t x}^{b_{m}}\left(\vartheta_{m}, \varphi_{m}\right)$ & $54.0 \mathrm{~dB}$ \\
\hline$L_{(\text {sat }, \text { out })}^{r t n, d w n}$ & $1.75 \mathrm{~dB}$ \\
\hline$L_{\text {sat,ant }}^{r t n, d w n}$ & $1.0 \mathrm{~dB}$ \\
\hline$L_{\text {sat }, O B O}^{r t n, d w n}$ & $4.0 \mathrm{~dB}$ \\
\hline$G_{f S a t, r x}^{b_{m}}\left(\vartheta_{m}, \varphi_{m}\right)$ & $54 \mathrm{~dB}$ \\
\hline$L_{\text {sat,ant }}^{\text {fwd,up }}$ & $1.0 \mathrm{~dB}$ \\
\hline$T_{\text {sat }, \text { feeder }}$ & $691.83 \mathrm{~K}$ \\
\hline$N_{\text {fwd,up }}^{\text {othersys }}$ & $-207 \mathrm{~dB} / \mathrm{Hz}$ \\
\hline$\left.\left(\frac{C}{I}\right)_{f w d, u p}^{I M}\right)$ & $27.0 \mathrm{~dB}$ \\
\hline \multicolumn{2}{|l|}{ GW } \\
\hline$P_{G W, t x}^{\max }$ & $8.97 \mathrm{dBW}$ \\
\hline$G_{G W, t x}^{\max }$ & $65.2 \mathrm{~dB}$ \\
\hline$L_{G W, t x}^{o u t}$ & $2.0 \mathrm{~dB}$ \\
\hline$L_{G W, t x}^{\text {pointing }}$ & $1.1 \mathrm{~dB}$ \\
\hline$L_{G W, t x}^{O B O}$ & $6 \mathrm{~dB}$ \\
\hline$G_{G W, r x}^{\max }$ & $61.5 \mathrm{~dB}$ \\
\hline$T_{G W}$ & $290 \mathrm{~K}$ \\
\hline$I_{r t n, d w n}^{A C I}$ & $10 \%$ incr. to noise \\
\hline$\left(\frac{C}{I}\right)_{r t n, d w n}^{I M}$ & $22.0 \mathrm{~dB}$ \\
\hline
\end{tabular}

